\title{
Identification of a novel mutation in the EXT1 gene from a patient with multiple osteochondromas by exome sequencing
}

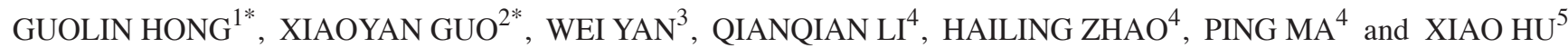 \\ ${ }^{1}$ Department of Laboratory Medicine, The First Affiliated Hospital of Xiamen University, Xiamen, Fujian 361003; \\ Departments of ${ }^{2}$ Laboratory Medicine and ${ }^{3}$ Bone Tumors, The Fuzhou Second Affiliated Hospital of Xiamen \\ University, Fuzhou, Fujian 350007; ${ }^{4}$ Medical College, Xiamen University, Xiamen, Fujian 361102; \\ ${ }^{5}$ Shenzhen Huada Gene Research Institute, Shenzhen, Fujian 518083, P.R. China
}

Received October 20, 2015; Accepted November 18, 2016

DOI: $10.3892 / \mathrm{mmr} .2016 .6086$

\begin{abstract}
Multiple osteochondromas (MO) is an autosomal skeletal disease with an elusive molecular mechanism. To further elucidate the genetic mechanism of the disease a three-generation Chinese family with MO was observed and researched, and a novel frameshift mutation (c.335_336insA) in the exotosin 1 (EXT1) gene of one patient with MO was observed through exome sequencing. This was further validated by Sanger sequencing and comparison with 200 unrelated healthy controls. Immunohistochemistry and multiple sequence alignment were performed to determine the pathogenicity of the candidate mutation. Multiple sequence alignment suggested that codon 335 and 336 in the EXT1 gene were highly conserved regions in vertebrates. Immunohistochemistry revealed that EXT1 protein expression levels were decreased in a patient with $\mathrm{MO}$ and this mutation compared with a patient with MO who had no EXT1 mutation. Owing to the appearance of c.335_336insA in exon 1 of EXT1, a premature stop codon was introduced, resulting in truncated EXT1. As a result integrated and functional EXT1 was reduced. EXT1 is involved in the biosynthesis of heparan sulfate (HS), an essential molecule, and its dysfunction may lead to MO. The novel mutation of c.335_336insA in the EXT1 gene reported in the present study has enlarged the causal mutation spectrum of $\mathrm{MO}$, and may assist genetic counseling and prenatal diagnosis of $\mathrm{MO}$.
\end{abstract}

Correspondence to: Dr Guolin Hong, Department of Laboratory Medicine, The First Affiliated Hospital of Xiamen University, 55 Zhenghai Road, Xiamen, Fujian 361003, P.R. China

E-mail: xmhg19899@sina.com

*Contributed equally

Key words: multiple osteochondromas, EXT1 gene, mutation, exome sequencing

\section{Introduction}

Multiple osteochondromas (MO), formerly referred to as hereditary multiple exostoses, is an autosomal dominant inherited disease with a recorded prevalence of 1/50,000 in Washington, USA, in 1994 (1). MO commonly presents as a benign bone tumor, however, 1-2\% of patients with MO progress into osteosarcoma or chondrosarcoma $(2,3)$. MO typically manifests as abnormalities in skeletal development, primarily involving long bones and the knees, ankles, elbows, wrists, shoulders and pelvis. Bony prominences of ranging size typically emerge around the epiphysis of long bones in the limbs of patients with MO $(4,5)$. Osteochondromas normally emerge during infancy and tend to present with related symptoms during adolescence, as the number and size of tumors often increases with age until growth terminates (6). Owing to the expanding tumors, patients with MO often suffer from pain, nerve and vascular compression, joint deformity and limitation of activity among other symptoms, including a shortened stature $(7,8)$.

Although the molecular mechanisms underlying MO are yet to be fully clarified, previous studies have reported that the exotosin (EXT) family is responsible for MO (9-11). EXT genes encode proteins that are involved in the biosynthesis of heparan sulfate (HS), an essential molecule in the growth and development of normal cells and dysfunction of EXT genes may lead to MO (12,13). EXT1 (14), EXT2 (15) and EXT3 (16) are the most important members of the EXT family (17). A common genetic basis exists in $90 \%$ of $\mathrm{MO}$ cases: Mutations in EXT1 or EXT2 trigger an early introduction of the termination codon and corresponding partial or total EXT gene deletion, resulting in a loss of protein function. $80 \%$ of EXT mutations are nonsense, frameshift or splicing mutations, with large fragment deletions and insertions rarely reported $(18,19)$.

With the development of DNA sequencing technology, gene mapping of diseases via exome sequencing has emerged, exhibiting the advantage of covering all exon regions. Exome sequencing has already been applied in the research of tumors and genetic diseases, through the screening of novel and pathogenic genetic variants of all exons or targeted sequences $(20,21)$. 
In the present study, exome sequencing was performed on a patient from a three-generation Han Chinese family with hereditary MO. Data were filtered from the 1000 Genome Project (http://www.1000genome.org), the Single Nucleotide Polymorphism (dbSNP) database for Chinese Han SNP (build 132; http://www.ncbi.nlm.nih.gov/project/SNP/) and other databases. Sanger sequencing of the MO patient, an unaffected relative and 200 unrelated healthy controls further validated the candidate mutation. For further confirmation, four individuals from an unrelated family with MO were examined. In addition, immunohistochemistry and multiple sequence alignment were performed to appraise the importance of the identified causal gene mutation.

\section{Materials and methods}

Human subjects. A Han Chinese family with MO from Fujian Province, China, was included in the present study (Table I; Fig. 1A). The proband (II4) was a 27 year old man who had displayed multiple, expanding exostoses for twenty years. Another family with MO (affected individuals: II2, II3 and II4; unaffected individual: II1) lacking an EXT1 gene mutation were included for further validation (Fig. 1B). 200 unrelated healthy subjects (100 males and 100 females, aged 10-40 years) with the same geographical ancestry were included as controls. Clinical data of all subjects was carefully recorded by the resident, with all participants receiving a general examination that included a computed radiography examination of long bones, truncal and acral joints by two experienced, independent orthopedic physicians. $5 \mathrm{ml}$ venous blood was drawn from 2 family members and the 200 healthy controls using EDTA-K2 anticoagulant vacuum blood vessels. The present study was approved by the Ethics Committee of The Fuzhou Second Affiliated Hospital of Xiamen University. All subjects provided signed, informed consent prior to participation.

Exome sequencing. Genomic DNA of the subjects was extracted from peripheral blood samples, using the QIAamp DNA Blood Midi kit (Qiagen GmbH, Hilden, Germany) according to the manufacturer's protocols. The extracted DNA was subsequently sent to the Huada Gene Research Institute (Shenzhen, China) for the following experiments, including exome sequencing. Initially, a total of 49 genes implicated in genetic tumors (Table II) were selected for exome sequencing, which was based on the gene capture strategy from the Huada Gene Research Institute (22). For exome sequencing, $3 \mu \mathrm{g}$ of extracted DNA from the patient with MO (II4) was randomly sheared into 100-200 bp fragment libraries by the Covaris LE220 ultrasonoscope (Covaris, Inc., Woburn, MA, USA), and purified by AMPure beads (Beckman Coulter, Inc., Brea, CA, USA). The purified DNA fragment tails were repaired and ' $A$ ' adapters were ligated to both ends of the resulting fragments which carried specific ' $\mathrm{T}$ ' linkers. In this way, the cDNA library was constructed completely. Prepared DNA was then amplified by ligation-mediated polymerase chain reaction (LM-PCR), purified, and hybridized to the NimbleGen Human custom array (NimbleGen; Roche Molecular Diagnostics, CA, USA) for enrichment. Reaction conditions were as follows: 1 cycle of $98^{\circ} \mathrm{C}$ for $30 \mathrm{sec}, 15$ cycles of $98^{\circ} \mathrm{C}$ for $10 \mathrm{sec}, 60^{\circ} \mathrm{C}$ for $30 \mathrm{sec}$ and $72^{\circ} \mathrm{C}$ for $30 \mathrm{sec} ; 1$ cycle of $72^{\circ} \mathrm{C}$ for $5 \mathrm{~min}$. Non-hybridized fragments were subsequently washed out. Both non-captured and captured LM-PCR products were subjected to an Eva Green (3100; Biotium, Inc., Hayward, CA, USA) Real-Time Fluorescence Quantitative PCR System (StepOne; Applied Biosystems; Thermo Fisher Scientific, Inc., Waltham, MA, USA) to estimate the magnitude of enrichment based on previous work (23). Each captured library was subsequently loaded onto the Hiseq2000 platform (Illumina, Inc., San Diego, CA, USA), and high-throughput sequencing was performed for each captured library independently to ensure that each sample met the desired average fold-coverage. Raw image files were processed by Illumina base calling software 1.7 (Illumina, Inc.) and the sequences of each individual were generated as $90 \mathrm{bp}$ paired-end reads.

Read mapping and variant analysis. Raw read sequencing quality was assessed following completion of exome sequencing, to ensure that low quality and contaminated reads were removed. Reads with too many $\mathrm{N}$ bases $(>10 \%)$ or low base quality $(>50 \%$ bases with base quality $<5)$ were discarded. Burrows-Wheeler Aligner (BWA) software (version hg19, build 37.1, http://bio-bwa.sourceforge.net/) was then used to align clean reads to the (University of California Santa Cruz (UCSC) human reference genome. Evaluation of the capture experiment was executed at the same time. Based on the BWA alignment results, Short Oligonucleotide Analysis Package snp software (version 1.03; http://soap.genomics.org.cn/index. html) and SAMtools software (version 0.9.41; http://sihua .us/samtools.htm) were used to search for single nucleotide variant (SNV) and insertion and deletion (indel) mutations, respectively. Once base polymorphisms of the target region were obtained, information of interest was aligned to data from the following databases by the Huada Gene Research Institute: the National Center for Biotechnology Information Database of Short Genetic Variation (dbSNP) (https://www.ncbi.nlm. nih.gov/snp/), the International HapMap Project (ftp://ftp .ncbi.nlm.nih.gov/hapmap/), the 1000 Genomes Project (ftp://www.1000genome.org), the Exome Sequencing Project (ESP; esp6500siv2), the Exome Aggregation Consortium (ExAC; version 0.3) and the Kaviar database (version no. $160,204)(24)$, which include various ethnic groups. Mutations of interest were screened, marked and analysed for gene function. Quality control, carried out before analysis for clean data and after data processing, was present throughout this pipeline in order to obtain clean data and alignments.

Sanger sequencing. Confirmatory Sanger sequencing was carried out in the proband and his sister (II5) to validate the candidate mutation that was identified by targeted exome sequencing. Target sequences from 200 unrelated healthy controls also underwent Sanger sequencing to estimate population frequencies and the pathogenicity of the candidate mutation. Sanger sequencing was performed using the standard protocol (18). Primers used were as follows: 5'-CAG TCCGGATCATTTCTGGCC-'3 and 5'-ACTGAGGTGACA ACTGGTCTC-'3. Sequence comparisons and analyses were performed using DNAman 8.0 (Lynnon Biosoft, San Ramon, CA, USA) and Chromas 2.0 (Technelysium Pty, Ltd., South Brisbane, Australia), respectively. 
Table I. Characteristics of family members observed in the present study.

\begin{tabular}{lclll}
\hline Subject & Age (years) & Sex & Multiple osteochondromas & Participation in present study \\
\hline II4 & 27 & Male & Affected & Yes \\
II5 & 31 & Female & Unaffected & Yes \\
II6 & 28 & Male & Affected & No \\
II7 & 23 & Female & Affected & No \\
III9 & 3 & Female & Affected & No \\
\hline
\end{tabular}

Table II. Genes captured in the present study.

\begin{tabular}{|c|c|c|c|}
\hline Chromosome & Gene symbol & Chromosome & Gene symbol \\
\hline Chr1 & $\begin{array}{l}\text { SDHB; MUTYH; NTRK1; } \\
\text { SDHC; CDC73; FH }\end{array}$ & Chr12 & CDKN1B; CDK4 \\
\hline Chr2 & $\begin{array}{l}\text { EPCAM; MSH2; MSH6; } \\
\text { TMEM127; PMS1; BARD1 }\end{array}$ & Chr13 & BRCA2; RB1 \\
\hline Chr3 & VHL; MLH1 & Chr14 & MAX; MLH3 \\
\hline Chr5 & APC; RAD50 & Chr16 & PALB2; CDH1 \\
\hline Chr7 & PMS2; MET & Chr17 & $\begin{array}{l}\text { TP53; FLCN; NF1; BRCA1; } \\
\text { RAD51C; BRIP1; AXIN2 }\end{array}$ \\
\hline Chr8 & NBN; EXT1 & Chr18 & SMAD4 \\
\hline Chr9 & CDKN2A & Chr19 & STK11 \\
\hline Chr10 & RET; BMPR1A; PTEN & Chr22 & CHEK2; NF2 \\
\hline Chr11 & $\begin{array}{l}\text { EXT2; SDHAF2; MEN1; } \\
\text { MRE11A; ATM; SDHD }\end{array}$ & Total & 49 genes \\
\hline
\end{tabular}

Histochemistry and immunohistochemistry. Chondroma tissues from the proband and an unrelated patient with MO who lacked the EXT1 gene mutation, were fixed with $1 \%$ paraformaldehyde for $24 \mathrm{~h}$ at $4^{\circ} \mathrm{C}$, rinsed with phosphate buffered saline (PBS), decalcified by immersion in $10 \%$ nitric acid and formaldehyde overnight and embedded in paraffin. Paraffin-embedded chondroma tissues were sectioned (5 $\mu \mathrm{m}$ thick) and placed on glass slides. For histochemistry, the tissue slides were dewaxed in xylene, hydrated with graded ethanol, and stained by toluidine blue and hematoxylin-eosin, for $5 \mathrm{~min}$ for each at room temperature and viewed with a fluorescence microscope (Olympus Corporation, Tokyo, Japan) at x40 magnification).

For immunohistochemistry, based on the standard procedure of high sensitivity two-step detection kit (cat. no. PV-9001; ZSGB-BIO, Beijing, China), dewaxed and hydrated chondroma sections were treated with $3 \%$ hydrogen peroxide solution for $10 \mathrm{~min}$, rinsed with PBS and incubated with rabbit polyclonal anti-EXT1 antibody (cat. no. ab177101; 1:50 working dilution; Abcam, Cambridge, UK) at $4^{\circ} \mathrm{C}$ overnight. Chondroma sections were treated with Polymer Helper (an assistant that helps the macromolecular detection system combine with the detected primary antibody molecule with higher sensitivity, included with the two-step detection kit) for 15 min, rinsed with PBS and incubated with the secondary antibody (cat. no. K152709C, polymer of anti-rabbit IgG; OriGene Technologies, Inc.) at $37^{\circ} \mathrm{C}$ for $15 \mathrm{~min}$ and stained using the DAB substrate kit (Vector Laboratories, Inc., Burlingame, CA, USA) and Mayer's hematoxylin, and viewed with an fluorescence microscope (Olympus Corporation) at x15 magnification. Targeted amino acid sequences of the primary antibody for EXT1 were 5-162 of human EXT1, a region upstream of the novel mutation.

\section{Results}

Clinical imaging and pathological examination. Specialized examination revealed that the proband had a valgus deformity in the left knee, and prominent tumors of ranging size in the arms, knees and pelvis. Exogenous tumors emerged from the distal end of bilateral femurs, the proximal end of bilateral tibias and fibulae, and around the pelvis (Fig. 1C-E), partially manifesting as irregular cauliflowers of varying size, with calcific cartilage caps. The tumors were connected to the wide base of the parent bone.

Tissue structure of MO was visualized by staining (Fig. 1F), revealing that layers of fibrous perichondrium were present around the cartilage cap, in which there was a typical cluster of chondrocytes growing neatly.

Exome sequencing and validation of Sanger sequencing. Exome sequencing of 49 genes linked to hereditary tumors was performed for the subject. For the targeted regions, the mean sequencing depths of $>80 \%$ of the 49 genes were yielded 

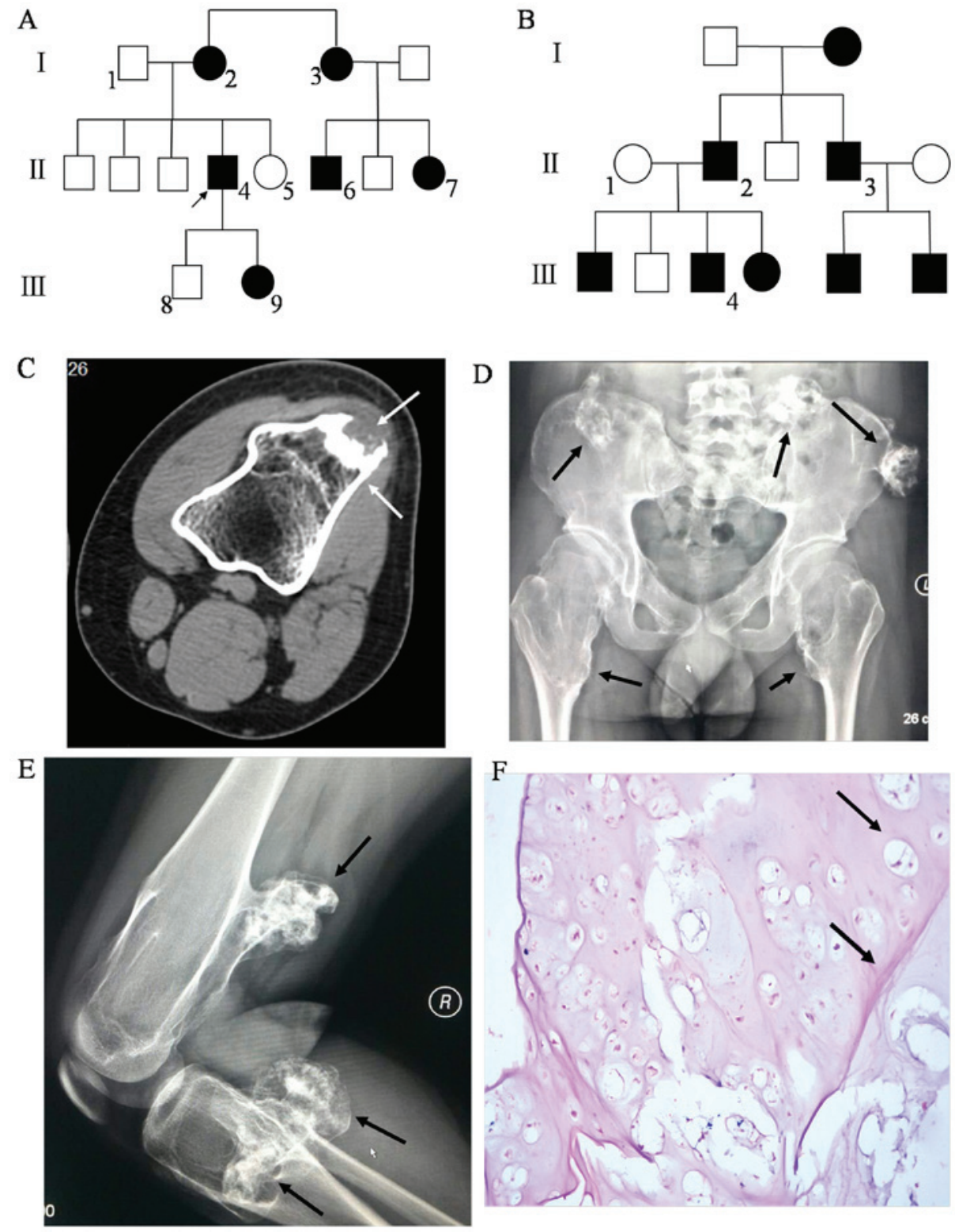

Figure 1. Pedigree and clinical characteristics of the proband with MO. (A) Pedigree of the family with MO observed in the present study. Black arrow indicates the proband. (B) Pedigree of an unrelated family with MO. (C-E) MO growing in the back of the articular surface of either end of the femur (indicated by white arrows in $\mathrm{C}$, the lower black arrows in $\mathrm{D}$ and the upper black arrow in E), the hipbones of the pelvis (indicated by the upper black arrows in D), and the upper part of the tibia and fibula of the proband (indicated by the lower black arrows in E). (F) Hematoxylin and eosin staining of the osteochondromas tissue from the proband. The uppermost arrow indicates the typical and areatus chondrocytes growing in the perichondrium, and the lower arrow indicates the fibroid perichondrium distributing layer by layer in the outer tissue. Viewed under 40x magnification. MO, multiple osteochondromas.

$>100$ times. The mean coverage of targeted exons for $>10$ reads was $92.46 \%$ and for $>20$ reads $89.82 \% .289$ SNVs and 36 indels were identified within the captured and flanking regions, including 21 nonsynonymous SNVs, 2 indels in coding exons and splicing mutations in introns (Table III). Considering the alteration of gene function caused by the mutations and their respective population frequencies, a frameshift mutation (c.335dupA) in EXT1 remained as candidate for causal mutations, further validated by Sanger sequencing of the patient with MO patient, the unaffected sister and 200 healthy controls. The heterozygous insertion (c.335_336insA) in EXT1 was identified as a candidate pathogenic gene mutation, as it was present in the proband (Fig. 2A) but absent in the unaffected individual (Fig. 2B) and the 200 healthy controls, as well as an unrelated family with MO. Multiple sequence alignment revealed that codon 335 and codon 336 of EXT1 are highly conserved in vertebrate species with a relatively close relationship to humans (Macaca mulatta, Pan troglodytes and Pongo abelii) and species with a remote relationship to humans (Mus musculus, Rattus norvegicus) (Fig. 2C). This demonstrates its functional importance.

Histochemical staining and immunohistochemistry. To evaluate the functional impact of c.335_336insA on the EXT1 protein, protein expression levels of EXT1 in two unrelated families with $\mathrm{MO}$ were detected using a supersensitive two-step immunohistochemistry method. EXT1 protein expression levels in the proband patient with $\mathrm{MO}$ and the c.335_336ins A mutation (Fig. 3A) were markedly decreased compared with those in a patient with MO that lacked any EXT1 mutation (Fig. 3B). 
Table III. Nonsynonymous single nucleotide variants and indels identified from targeted exome resequencing.

\begin{tabular}{|c|c|c|c|c|c|c|}
\hline Gene & Mut_type & Intron/Exon & Mutation & Function & Freq_1000g2015aug & esp6500siv2 \\
\hline MUTYH & Het & Exon12 & $\mathrm{C}>\mathrm{G}$ & nonsynonymous & 0.313498 & 0.2541 \\
\hline EPCAM & Hom & Exon3 & $\mathrm{T}>\mathrm{C}$ & nonsynonymous & 0.666134 & 0.5667 \\
\hline MSH6 & Het & Exon1 & $\mathrm{G}>\mathrm{A}$ & nonsynonymous & 0.200879 & 0.1796 \\
\hline MSH6 & Het & Exon $2 / 4$ & $\mathrm{C}>\mathrm{T}^{\mathrm{a}}$ & nonsynonymous & - & 0.000077 \\
\hline APC & Hom & Exon14/16/17 & $\mathrm{T}>\mathrm{A}$ & nonsynonymous & 0.865415 & 0.8263 \\
\hline PMS2 & Het & Exon11 & $\mathrm{T}>\mathrm{C}$ & nonsynonymous & 0.883187 & 0.8704 \\
\hline PMS2 & Het & Exon11 & $\mathrm{G}>\mathrm{T}$ & nonsynonymous & 0.112021 & 0.0335 \\
\hline MET & Het & Exon2 & $\mathrm{A}>\mathrm{G}$ & nonsynonymous & 0.0329473 & 0.0146 \\
\hline $\mathrm{NBN}$ & Het & Exon5/6 & $\mathrm{C}>\mathrm{G}$ & nonsynonymous & 0.357029 & 0.2866 \\
\hline BMPR1A & Het & Exon3 & $\mathrm{C}>\mathrm{A}$ & nonsynonymous & 0.4998 & 0.3895 \\
\hline MEN1 & Het & Exon10/11 & $\mathrm{T}>\mathrm{C}$ & nonsynonymous & 0.834465 & 0.9084 \\
\hline ATM & Hom & Exon40 & $\mathrm{A}>\mathrm{G}$ & nonsynonymous & 1 & - \\
\hline BRCA2 & Het & Exon10 & $\mathrm{A}>\mathrm{C}$ & nonsynonymous & 0.249401 & 0.2332 \\
\hline BRCA2 & Hom & Exon14 & $\mathrm{T}>\mathrm{C}$ & nonsynonymous & 0.975839 & 0.9777 \\
\hline MLH3 & Het & Exon5 & $\mathrm{C}>\mathrm{T}$ & nonsynonymous & 0.00459265 & 0.000077 \\
\hline MLH3 & Hom & Exon2 & $\mathrm{T}>\mathrm{C}$ & nonsynonymous & 0.990415 & 0.9888 \\
\hline TP53 & Hom & Exon4 & $\mathrm{G}>\mathrm{C}$ & nonsynonymous & 0.542931 & 0.63 \\
\hline FLCN & Hom & Exon8 & $\mathrm{C}>\mathrm{T}$ & nonsynonymous & 0.0996406 & 0.0775 \\
\hline FLCN & Het & Exon8 & $\mathrm{C}>\mathrm{T}^{\mathrm{b}}$ & nonsynonymous & - & - \\
\hline AXIN2 & Het & Exon6 & $\mathrm{G}>\mathrm{A}$ & nonsynonymous & 0.00359425 & - \\
\hline AXIN2 & Het & Exon2 & $\mathrm{G}>\mathrm{A}$ & nonsynonymous & 0.33766 & 0.3948 \\
\hline EXT1 & Het & Exon1 & $\operatorname{ins}^{c}$ & Indel & - & - \\
\hline PTEN & Hom & Exon $1 / 2$ & delT & Indel (splicing) & 1 & - \\
\hline
\end{tabular}

${ }^{\mathrm{a}} \mathrm{Gene}$ frequency from other databases: Kaviar_AF(0.0000065); ExAC_AFR(0.00009739); ExAC_Freq(0.000008236). ${ }^{\mathrm{b}} \mathrm{Gene}$ frequency from other databases: ExAC_Freq(0.000008238); ExAC_EAS(0.0001); Kaviar_AF(0.0000065); ${ }^{\circ}$ No database had relevant frequency reports for the mutation. Het, heterozygous; Hom, homozygous; indel, insertion or deletion.
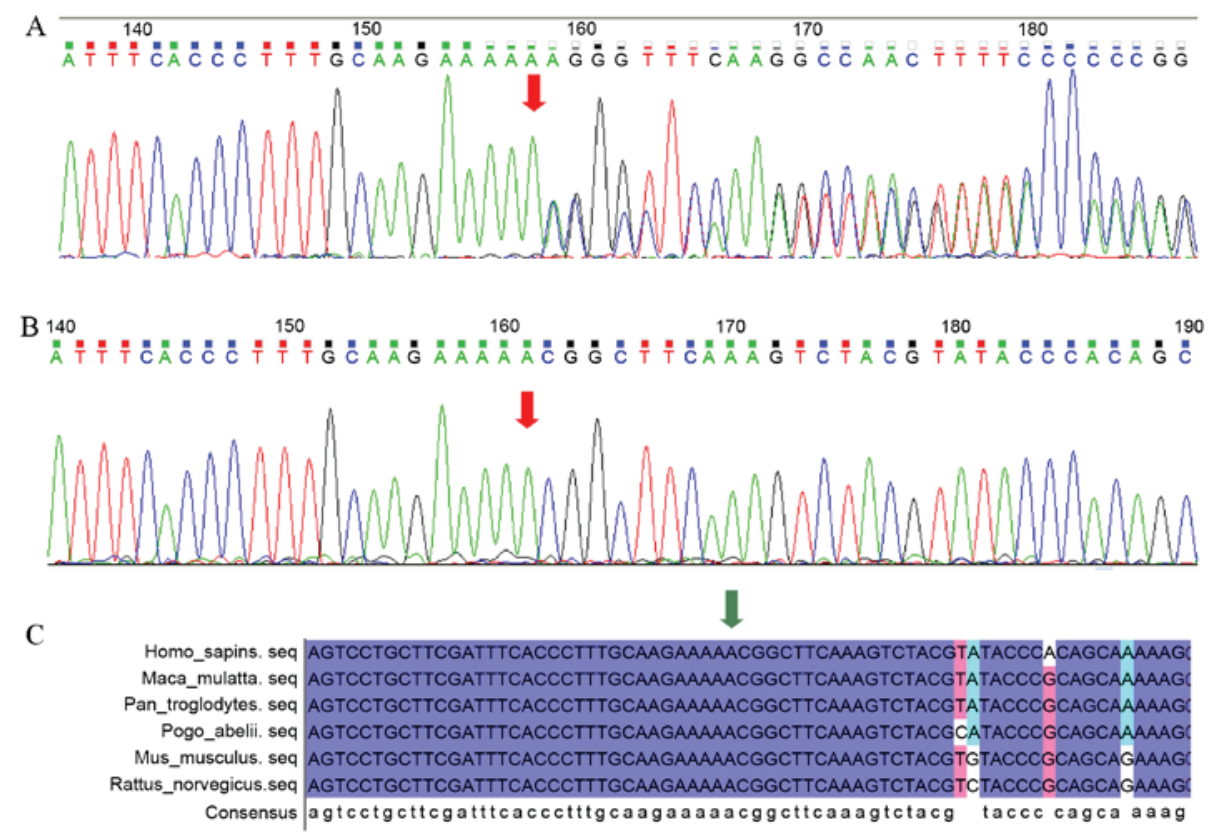

Figure 2. Identification of a novel frameshift mutation in codon 336 of the EXT1 gene. (A) A novel insertion mutation was detected in the EXT1 gene by Sanger sequencing in the proband. The red arrow denotes the position of the mutation. (B) No mutation was detected in the same position in the unaffected subject, II5. (C) Multiple alignment of part sequences of exon 1 of EXT1, spanning codon 335 and 336 between various vertebrate species (the green arrow indicates the location of c.335_336insA). EXT, exotosin. 

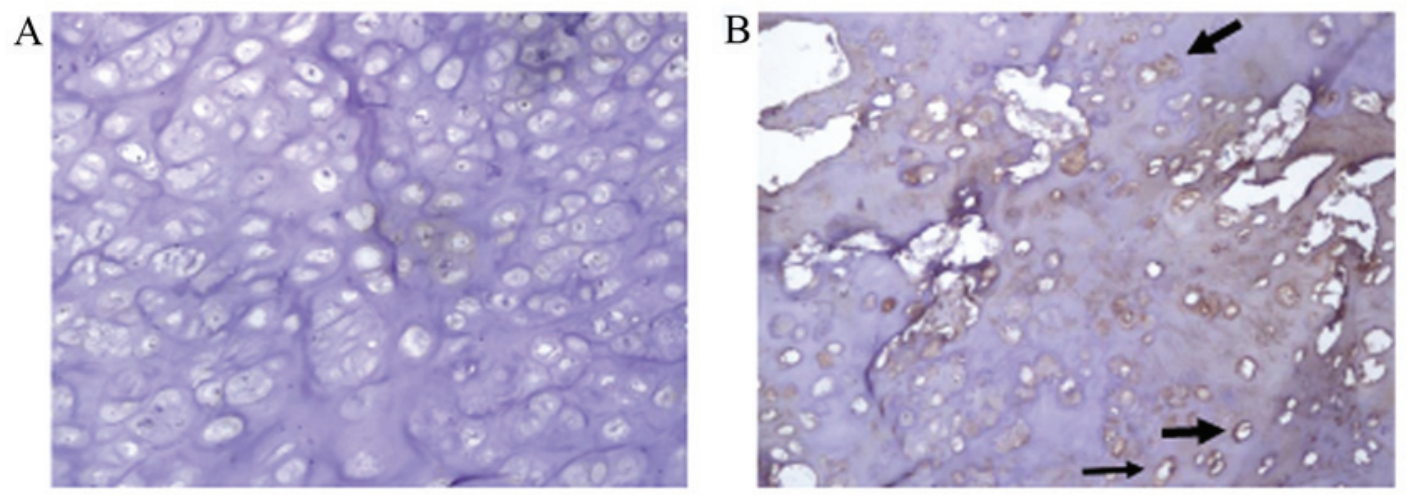

Figure 3. Immunohistochemical screening of chondrocytes. (A) Immunohistochemical staining of EXT1 in chondrocytes from the superficial layers of cartilage caps of the proband. (B) Another patient with multiple osteochondromas from an unrelated family that lacks an EXT1 mutation. Black arrowheads represent a chondrocyte with functional EXT1. Viewed under x15 magnification. EXT, exotosin.

A

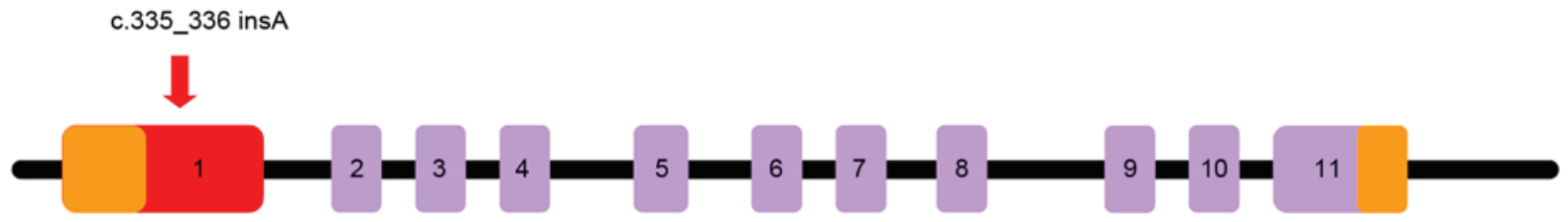

B

Normal exostosin-1

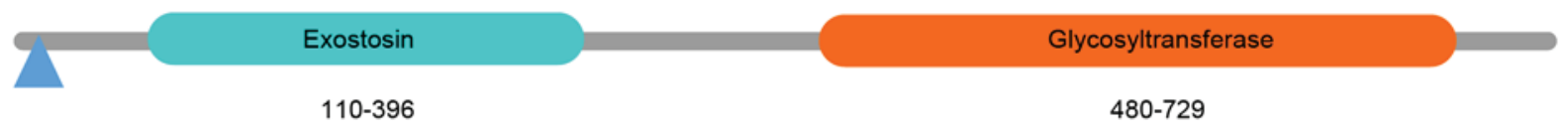

$\mathrm{C}$

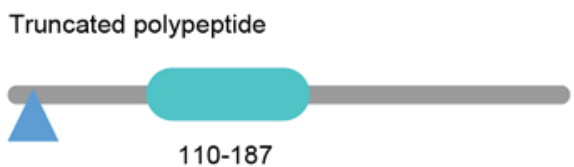

Figure 4. (A) EXT1 gene structure. c.335_336insA was discovered in exon 1 (denoted by red arrow). (B) Functional domains of the EXT1 protein encoded by the normal EXT1 gene. The exostosin domain contains 287 amino acids (110 to 396), and the glycosyltransferase domain contains 250 amino acids (480 to 729). (C) The truncated polypeptide that results from the mutation.

\section{Discussion}

To date, 196 different mutations distributed in the exon/intron 1 of the EXT1 gene have been identified from more than 200 families with MO, according to the Multiple Osteochondromas Mutation Database (version 2.0, http://medgen.ua.ac.be/LOVDv.2.0/home.php). EXT1 is comprised of 11 constructive exons (Fig. 4A) encoding 746 amino acids and possesses two domains: exostosin and glycosyltransferase (Fig. 4B). The mutation identified in the present study, c.335_336insA, appeared between codon 335 and codon 336 in exon 1 of EXT1 (Fig. 4A), resulting in a frameshift mutation in the first domain of the EXT1 gene and creating a premature stop codon at amino acid 187 (Fig. 4C). This resulted in the loss of part of the exostosin domain and the entire glycosyltransferase domain, producing a truncated polypeptide (Fig. 4C).
As a putative tumor-suppressor in the EXT gene family, EXT1 mutations have been suggested to be a major cause of MO, in addition, patients with MO who have EXT1 gene mutations tend to display more severe symptoms and suffer a greater risk for malignant transformation than patients with EXT2 gene mutations (25-27). EXT1 and EXT2 proteins form a stable complex as a glucuronic acid sugar-based transfer enzyme and $\mathrm{N}$-acetylglucosamine glycosyltransferase, catalyzing the polymerization of HS chains in the endoplasmic reticulum and Golgi apparatus (28). HS is ubiquitously expressed on the cell surface, and as a component of extracellular matrix glycoprotein, it is involved in the mediation of cell adhesion, signal transduction and the receptor ligand binding process (29). Previous studies have indicated that HS is an essential molecule for the growth and differentiation of normal chondrocytes and its dysfunction may be associated with MO (30-33). 
In the present study a novel frameshift mutation in exon 1 of EXT1 gene, c.335_336insA, was identified in one affected individual of a family with MO and was not identified in either an unaffected family member or in any of 200 unrelated healthy controls. It was also not present in individuals from an unrelated family with MO. Multiple sequence alignment and immunohistochemistry both confirmed the pathogenicity of the c.335_336insA mutation. Furthermore, according to the number and distribution of the affected patients in the family with MO (Fig. 1A), it is possible to infer that the c.335_336insA mutation originated from the mother of the proband and was transmitted to the daughter of the proband in an autosomal dominant manner.

The c.335_336insA mutation is located within the exostosin domain of the EXT1 gene, and results in a frameshift at codon 335 and a change of an asparagine to a lysine. The occurrence of a premature stop codon and truncated EXT1 usually triggers nonsense-mediated mRNA decay and reduces the level of functional EXT1 (34,35). Immunohistochemistry also confirmed that the EXT1 expression level was decreased. This result is consistent with previous studies, which demonstrated that truncated EXT1 fails to fold correctly with exostosin-2 and rapidly degrades, resulting in decreased EXT1 levels in the chondrocytes of patients with MO $(21,36)$.

Multiple alignment of the sequences spanning the mutation site suggested that this point of interest is extraordinarily ancient and conserved, with scarcely any differences in mammalian species either closely or distantly related to humans. Referring to evidence from previous studies, it is possible to infer that MO in the proband of the present study was caused by the c.335_336insA mutation, which directly resulted in the functional loss of the two important domains of EXT1. This hindered the effective folding of EXT1 and efficient biosynthesis of HS, leading to disruption of signal transduction. Ultimately, the proliferation and differentiation of chondrocytes escaped regulation and initiated the development of MO. Further studies are required to illuminate the molecular mechanisms underlying the actions of the two EXT1 domains, in particular the glycosyltransferase domain.

To conclude, a novel frameshift mutation c.335_336insA in EXT1 gene was discovered through exome sequencing and validated by Sanger sequencing. The pathogenicity of the mutation was further confirmed via multiple alignment and immunohistochemistry. The present study also emphasized the dysfunction of the EXT genes in the development of MO. This finding will aid early diagnosis and prenatal genetic screening of the remaining members in the studied family with MO.

\section{Acknowledgements}

The present study was supported by the National Natural Science Foundation of China (81371902), the Fujian Medical Innovation Project (2012-CX-30) and the Innovative Team Training Project in Fuzhou (2013- S-wt2).

\section{References}

1. Schmale GA, Conrad EU III and Raskind WH: The natural history of hereditary multiple exostoses. J Bone Joint Surg Am 76: 986-992, 1994.
2. Jennes I, Pedrini E, Zuntini M, Mordenti M, Balkassmi S, Asteggiano CG, Casey B, Bakker B, Sangiorgi L and Wuyts W: Multiple osteochondromas: Mutation update and description of the multiple osteochondromas mutation database (MOdb). Hum Mutat 30: 1620-1627, 2009.

3. Tian C, Yan R, Wen S, Li X, Li T, Cai Z, Du H and Chen H: A splice mutation and mRNA decay of EXT2 provoke hereditary multiple exostoses. PLoS One 9: e94848, 2014.

4. Stieber JR and Dormans JP: Manifestations of hereditary multiple exostoses. J Am Acad Orthop Surg 13: 110-20, 2005.

5. Jamsheer A, Socha M, Sowińska-Seidler A, Telega K, Trzeciak T and Latos-Bieleńska A: Mutational screening of EXT1 and EXT2 genes in Polish patients with hereditary multiple exostoses. J Appl Genet 55: 183-188, 2014.

6. Vanita V, Sperling K, Sandhu HS, Sandhu PS and Singh JR: Novel EXT1 and EXT2 mutations in hereditary multiple exostoses families of Indian origin. Genet Test Mol Biomarkers 13: 43-49, 2009.

7. Wu Y, Xing X, Xu S, Ma H, Cao L, Wang S and Luo Y: Novel and recurrent mutations in the EXT1 and EXT2 genes in Chinese kindreds with multiple osteochondromas. J Orthop Res 31: 1492-1499, 2013.

8. Jones KB, Datar M, Ravichandran S, Jin H, Jurrus E, Whitaker R and Capecchi MR: Toward an understanding of the short bone phenotype associated with multiple osteochondromas. J Orthop Res 31: 651-657, 2013.

9. Wuyts W and Van Hul W: Molecular basis of multiple exostoses: Mutations in the EXT1 and EXT2 genes. Hum Mutat 15: 220-227, 2000.

10. Wuyts W, Radersma R, Storm K and Vits L: An optimized DHPLC protocol for molecular testing of the EXT1 and EXT2 genes in hereditary multiple osteochondromas. Clin Genet 68: $542-547,2005$.

11. Lonie L, Porter DE, Fraser M, Cole T, Wise C, Yates L, Wakeling E, Blair E, Morava E, Monaco AP and Ragoussis J: Determination of the mutation spectrum of the EXT1/EXT2 genes in British Caucasian patients with multiple osteochondromas, and exclusion of six candidate genes in EXT negative cases. Hum Mutat 27: 1160, 2006.

12. McCormick C, Leduc Y, Martindale D, Mattison K, Esford LE, Dyer AP and Tufaro F: The putative tumour suppressor EXT1 alters the expression of cell-surface heparan sulfate. Nat Genet 19: 158-161, 1998.

13. Zak BM, Crawford BE and Esko JD: Hereditary multiple exostoses and heparan sulfate polymerization. Biochim Biophys Acta 1573: 346-355, 2002.

14. Ahn J, Lüdecke HJ, Lindow S, Horton WA, Lee B, Wagner MJ, Horsthemke B and Wells DE: Cloning of the putative tumour suppressor gene for hereditary multiple exostoses (EXT1). Nat Genet 11: 137-143, 1995.

15. Stickens D, Clines G, Burbee D, Ramos P, Thomas S, Hogue D, Hecht JT, Lovett M and Evans GA: The EXT2 multiple exostoses gene defines a family of putative tumour suppressor genes. Nat Genet 14: 25-32, 1996

16. Le Merrer M, Legeai-Mallet L, Jeannin PM, Horsthemke B, Schinzel A, Plauchu H, Toutain A, Achard F, Munnich A and Maroteaux P: A gene for hereditary multiple exostoses maps to chromosome 19p. Hum Mol Genet 3: 717-722, 1994.

17. Trebicz-Geffen M, Robinson D, Evron Z, Glaser T, Fridkin M, Kollander Y, Vlodavsky I, Ilan N, Law KF, Cheah KS, et al: The molecular and cellular basis of exostosis formation in hereditary multiple exostoses. Int J Exp Pathol 89: 321-331, 2008.

18. Pedrini E, De Luca A, Valente EM, Maini V, Capponcelli S, Mordenti M, Mingarelli R, Sangiorgi L and Dallapiccola B: Novel EXT1 and EXT2 mutations identified by DHPLC in Italian patients with multiple osteochondromas. Hum Mutat 26: $280,2005$.

19. Sarrión P, Sangorrin A, Urreizti R, Delgado A, Artuch R, Martorell L, Armstrong J, Anton J, Torner F, Vilaseca MA, et al: Mutations in the EXT1 and EXT2 genes in Spanish patients with multiple osteochondromas. Sci Rep 3: 1346, 2013.

20. Huang XF, Xiang P, Chen J, Xing DJ, Huang N, Min Q, Gu F, Tong Y, Pang CP, Qu J and Jin ZB: Targeted exome sequencing identified novel USH2A mutations in Usher syndrome families. PLoS One 8: e63832, 2013.

21. Liu H, Wu S, Duan L, Zhu W, Zhang S, Hu X, Jia W, Yang G, Liu C, Li W, et al: Identification of a novel EXT1 mutation in patients with hereditary multiple exostosis by exome sequencing. Oncol Rep 33: 547-552, 2015. 
22. Wu J, Matthaei H, Maitra A, Dal Molin M, Wood LD, Eshleman JR, Goggins M, Canto MI, Schulick RD, Edil BH, et al: Recurrent GNAS mutations define an unexpected pathway for pancreatic cyst development. Sci Transl Med 3: 92ra66, 2011

23. Livak KJ and Schmittgen TD: Analysis of relative gene expression data using real-time quantitative PCR and the 2(-Delta Delta C(T)) Method. Methods 25: 402-408, 2001.

24. Glusman G, Caballero J, Mauldin DE, Hood L and Roach JC: Kaviar: An accessible system for testing SNV novelty. Bioinformatics 27: 3216-3217, 2011.

25. Porter DE, Lonie L, Fraser M, Dobson-Stone C, Porter JR, Monaco AP and Simpson AH: Severity of disease and risk of malignant change in hereditary multiple exostoses. A genotype-phenotype study. J Bone Joint Surg Br 86: 1041-1046, 2004.

26. Francannet C, Cohen-Tanugi A, Le Merrer M, Munnich A, Bonaventure J and Legeai-Mallet L: Genotype-phenotype correlation in hereditary multiple exostoses. J Med Genet 38: 430-434, 2001.

27. Alvarez CM, De Vera MA, Heslip TR and Casey B: Evaluation of the anatomic burden of patients with hereditary multiple exostoses. Clin Orthop Relat Res 462: 73-79, 2007.

28. McCormick C, Duncan G, Goutsos KT and Tufaro F: The putative tumor suppressors EXT1 and EXT2 form a stable complex that accumulates in the Golgi apparatus and catalyzes the synthesis of heparan sulfate. Proc Natl Acad Sci USA 97: 668-4073, 2000

29. Esko JD and Lindahl U: Molecular diversity of heparan sulfate. J Clin Invest 108: 169-173, 2001.

30. Jones KB, Pacifici M and Hilton MJ: Multiple hereditary exostoses (MHE): Elucidating the pathogenesis of a rare skeletal disorder through interdisciplinary research. Connect Tissue Res 55: 80-88, 2014.
31. Koziel L, Kunath M, Kelly OG and Vortkamp A: Ext1-dependent heparan sulfate regulates the range of Ihh signaling during endochondral ossification. Dev Cell 6: 801-813, 2004

32. Anower-E-Khuda MF, Matsumoto K, Habuchi H, Morita H, Yokochi T, Shimizu K and Kimata K: Glycosaminoglycans in the blood of hereditary multiple exostoses patients: Half reduction of heparan sulfate to chondroitin sulfate ratio and the possible diagnostic application. Glycobiology 23: 865-876, 2013.

33. Jochmann K, Bachvarova V and Vortkamp A: Reprint of: Heparan sulfate as a regulator of endochondral ossification and osteochondroma development. Matrix Biol 35: 239-247, 2014.

34. Gerards M, van den Bosch B, Calis C, Schoonderwoerd K, van Engelen K, Tijssen M, de Coo R, van der Kooi A and Smeets H: Nonsense mutations in CABC1/ADCK3 cause progressive cerebellar ataxia and atrophy. Mitochondrion 10: 510-515, 2010

35. Inoue $\mathrm{K}$, Khajavi M, Ohyama $\mathrm{T}$, Hirabayashi $\mathrm{S}$, Wilson $\mathrm{J}$, Reggin JD, Mancias P, Butler IJ, Wilkinson MF, Wegner M and Lupski JR: Molecular mechanism for distinct neurological phenotypes conveyed by allelic truncating mutations. Nat Genet 36: 361-369, 2004.

36. Zhang F, Liang J, Guo X, Zhang Y, Wen Y, Li Q, Zhang Z, Ma W, Dai L, Liu X, et al: Exome sequencing and functional analysis identifies a novel mutation in EXT1 gene that causes multiple osteochondromas. PLoS One 8: e72316, 2013. 